\title{
ADAPTING CONWIP CHARACTERISTICS FOR Conventional Production Planning
}

\author{
GASTERMANN, B. C.; STOPPER, M. \& KATALinic, B.
}

Abstract: The main purpose of this paper is to introduce the reader to some characteristics of the Constant Work-In-Process (CONWIP) manufacturing system. Starting with basic principles of manufacturing like push and pull, an overview of the most common and significant production strategies and manufacturing systems is provided in order to allow a better understanding of the origins and properties of the CONWIP system. Being the focus of this paper, various aspects of CONWIP are discussed. Furthermore, by means of a simplified production order list, which is a CONWIP-based planning mechanism, the operating mode of CONWIP is demonstrated. Building upon this, a CONWIP implementation case study is presented by which aspects for conventional production planning are outlined.

Key words: CONWIP, supply chain management, optimization, production strategy
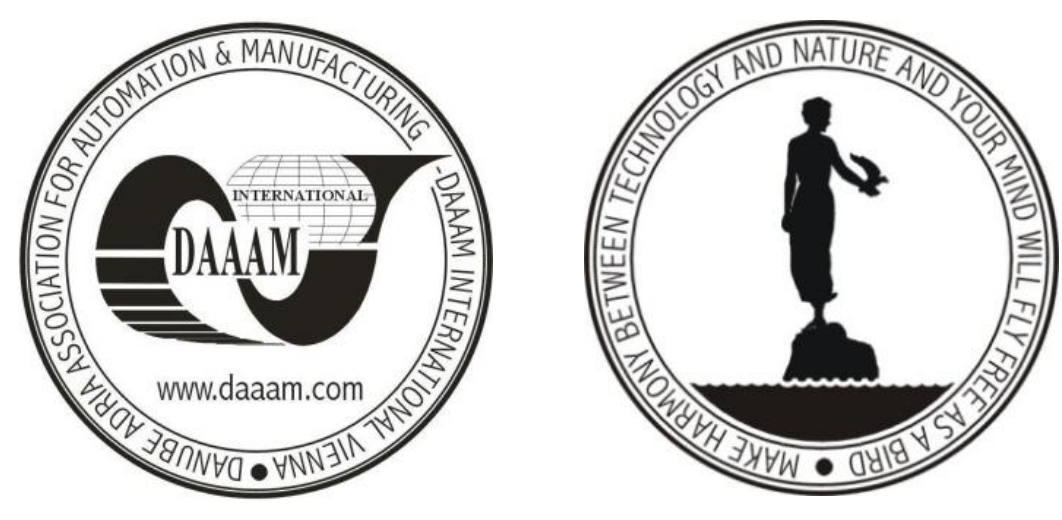

Authors' data: Dipl.-Ing. (FH) Gastermann, B[ernd] C[hristian]*, Prof. h.c. Dipl.Ing. Dr. Sc. Stopper, M[arkus]*, Univ.Prof. Dipl.-Ing. Dr.h.c.mult. Dr. Sc. Katalinic, B[ranko]**, *MKW® Austria, Industrial Research Center, Jutogasse 3, 4675, Weibern, Austria, **Vienna University of Technology, Karlsplatz 13, 1040, Vienna, Austria; bernd.gastermann@mkw.at, markus.stopper@ieee.org, katalinic@mail.ift.tuwien.ac.at

This Publication has to be referred as: Gastermann, B[ernd] C[hristian]; Stopper, M[arkus] \& Katalinic, B[ranko] (2012). Adapting CONWIP Characteristics for Conventional Production Planning, Chapter 46 in DAAAM International Scientific Book 2012, pp. 553-564, B. Katalinic (Ed.), Published by DAAAM International, ISBN 978-3-901509-86-5, ISSN 1726-9687, Vienna, Austria

DOI: $10.2507 /$ daaam.scibook.2012.46 


\section{Introduction}

Over the past several decades, the globalization of the manufacturing ecosystem has driven change, which impacted many companies around the world. Plenty of them benefited from the rapid globalization of industry and expansion of manufacturing. However, these also led to a change in the competitive environment of manufacturing companies. New requirements generate pressure on various aspects of production. For example, it becomes increasingly important to produce more in less time at even lower costs. This pressure caused manufacturing process designers to turn attention to the virtue of developing flexible and more efficient production planning and control (PPC) systems. According to (Spearman et al., 1990), such systems are those that "produce the right parts, at the right time, at a competitive cost". With that in mind, this paper will first discuss fundamentals and characteristics of some popular manufacturing control systems. The focus here, however, is on the Constant Work-In-Process (CONWIP) planning system, an approach that is rarely used within Europe. Eventually, a case study will be presented, which demonstrates the implementation of CONWIP for conventional production planning in a real production environment.

\section{Fundamentals of Production Planning and Control}

Before looking at concrete manufacturing system implementations, some fundamentals of PPC have to be explained. In this initiatory section, the basic principles of push and pull are explained. Furthermore, an approach to combine both of them to create a hybrid manufacturing system is shown. This will be important to understand for the implementation presented later in this paper.

\subsection{Push- and Pull-based Strategies}

Basically, there are two essential kinds of production strategies in supply chain management: Namely push- and pull-based systems. The use of these terms in conjunction with production control systems is very popular although there are no generally accepted definitions for them (Spearman et al., 1990).

Manufacturing systems that release work items according to a master production schedule (MPS) are classified as push-based systems. In such systems, production of goods is usually triggered by forecast demand and/or historic demand. Actual demand is ignored. Thus, schedules are generated that define the release of new production items. If new work has been released it is subsequently being processed at each step of the production line until it is eventually being stored at the finished goods inventory. Out of there, those goods are delivered to retailers and offered to customers. Given that, products are always fully produced in advance and sold as "anonymous" products, which means they are not related to actual consumer in any way until they are sold (Jodlbauer, 2008). During the manufacturing process in pushbased systems, internal states like capacities or work-in-process (WIP) are not being considered and do not affect the release of new work items. In other words, information and goods flow downstream the manufacturing chain from raw material 
inventory to finished goods inventory. Hence, push systems can inherently be described as "make-to-stock" (MTS) (see figure 1). With this build-ahead manufacturing approach, accuracy and reliability of demand forecasts are essential because they will prevent both excessive inventory levels and opportunity loss due to stock-out. Therefore, push-based systems are often used for high volume products where demand is stable and easily predictable.

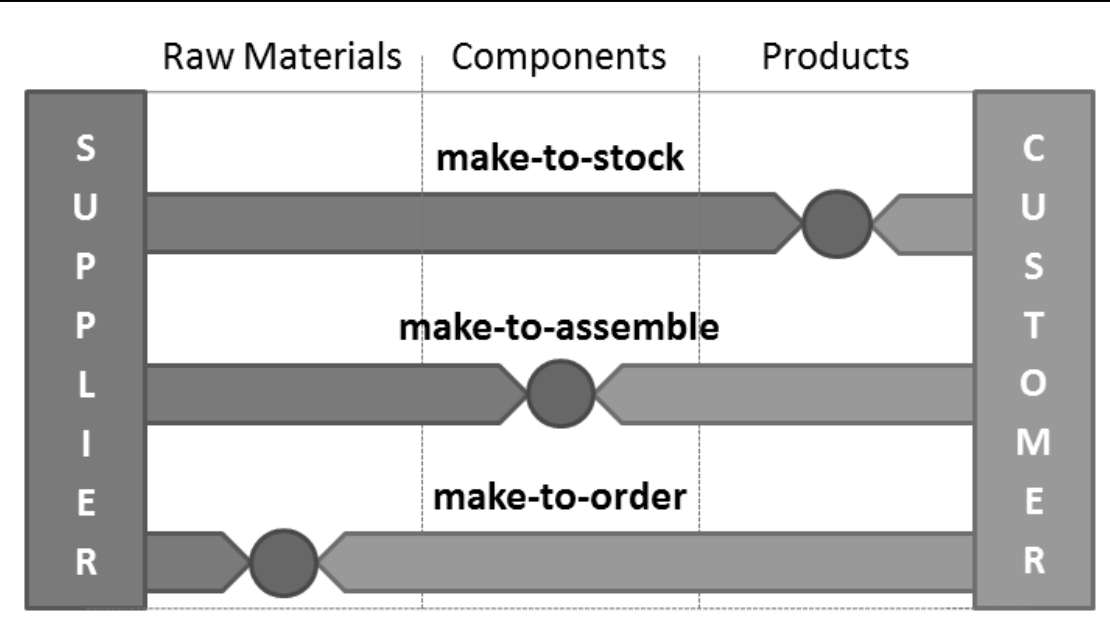

Fig. 1. This is a comparison of three different production approaches, each having a different Customer Order Decoupling Point (CODP)

Contrary to the push approach, pull-based production systems depend on actual demand, though it is irrelevant whether it derives from real customer demand or from internal demand of subsequent manufacturing stages. For example, production of goods is triggered as soon as demand is generated by the placement of a customer order. Likewise, the use of certain components by downstream work centres authorizes the start of production of even more of those components (Spearman et al., 1990). Dependence on actual demand inherently classifies these systems as "make-toorder" (MTO) (see figure 1). Authorization signals and other information required in this approach flow upstream because each manufacturing stage declares demand of goods from its precedent manufacturing stage. Furthermore, inventory and WIP levels are kept low by only producing exact amounts of a certain product at the time it is actually needed. Hence, problems of excessive inventory, which are quite common with the traditional MTS strategy, are relieved so that the amount of products in stock is lower while having more product types available (Jodlbauer, 2008). For this approach to work, it is important that lead time does not exceed the delivery date. Otherwise, MTO may create additional waiting time for the consumer to receive the product. Nonetheless, pull-based systems generally allow for more flexible customization. It is, therefore, most suitable for highly customized or customer-specific products of low volumes. Pull systems have several important advantages compared to other systems: Unit costs are kept low while high quality standards and low inventory levels can be maintained, which allows a reduction of necessary inventory space. Furthermore, the output stream of pull systems remains more steady and predictable. By avoiding to release work items too early (which 
keeps WIP at about the same low level), production flexibility is improved and floating capacity is encouraged. Pull systems deliberately establish a limit on WIP while push systems do not.

\subsection{Hybrid Production Systems}

When designing a manufacturing system, it is not always appropriate to adopt either the MTS or the MTO production approach. However, they are not mutually exclusive (Spearman et al., 1990), so it is possible to combine both of them into a hybrid production system, which is often referred to as "make-to-assemble" (MTA) (see figure 1). The term "assemble-to-order" (ATO) could also be used, although it is less common. Hybrid manufacturing strategies combine aspects of both push- and pull-based systems in the following way:

In a first step, basic components of a product are produced anonymously, that is, without a specific customer order (Jodlbauer, 2008), and stocked based on forecast demand. As soon as a customer order is placed, these stocked components are subsequently used to assemble the final customer-related product. Looking at this process, the inventory of components clearly marks the point that splits the manufacturing chain into MTS and MTO production. This point of transition from MTS to MTO is called the Order Penetration Point (OPP) (Olhager, 2003) or Customer Order Decoupling Point (CODP) (Jodlbauer, 2008). The actual position of this spot varies between manufacturing companies, dependent on the kind of approach they have decided to adopt. It is important to carefully select its location in order to gain benefits from both push and pull strategy. Moving the CODP closer to the customer, for example, improves responsiveness, while moving it farther away from the customer improves flexibility. Having the right balance, hybrid systems allow for higher order customization and flexibility as well as smaller lead times when compared to traditional MTS or MTO production (Olhager, 2003).

\section{Manufacturing Systems Overview}

Based upon these principles, various manual and electronic production control system implementations exist. Manufacturing companies usually try to adopt the one that fits their individual situations the most. In this chapter, some fundamental concepts of computer-assisted and manual production control systems are introduced in order to provide the reader with a general overview. It deals with some well-known variants, like Material Requirements Planning and Kanban. However, given the focus of this paper, Constant Work-In-Process (CONWIP) is dedicated a separate section in this paper where it will be discussed to a greater extent.

\subsection{Re-Order Point Planning}

Re-Order Point (ROP) Planning is a rather old but widely used method of inventory control, aiming to minimise total inventory holding costs and ordering costs. It is also used to automate inventory. The ROP represents a certain level of inventory, at which a signal is triggered to replenish that inventory (either from internal or external sources). Considering a certain lead time, the ROP must be high 
enough to allow ordered materials to arrive at the inventory before it is completely empty. Therefore, it is important to determine an appropriate level for the ROP. This decision is influenced by mainly two factors: The first one is the amount of stock that is expected to be consumed by production between placement of the replenishment order and arrival of ordered materials, which in turn could be influenced by delays like transportation or shortages. The second one is the safety stock, which is the minimum level of inventory that is held as protection against shortages and fluctuations in demand. The problem of this approach is that replenishment orders are derived from historic data and demand is expected to not show heavy fluctuations in a short timeframe.

\subsection{Material Requirements Planning}

Material Requirements Planning (MRP) is a very popular production planning and inventory control system, which is typically based on push strategy and can be used for various kinds of production tasks. It is able to plan manufacturing activities, delivery schedules, and purchasing activities. MRP also ensures that materials are available for production, as well as that finished products are available for delivery to customers. Maintaining the lowest possible material and product levels in stock is an important objective of the MRP system.

However, its major problem is integrity of data because errors in input data will eventually also generate incorrect output. Another problem is the fact that fixed lead times have to be specified, which will be assumed to be the same for each product, no matter how many items have to be produced or what other concurrent items are being made at that time (Spearman et al., 1990). This could lead to a rather pessimistic specification of lead times, resulting in high WIP and inventory levels. Furthermore, capacity is not taken into account, which could lead to implementation problems if there are any internal or external capacity constraints.

\subsection{Manufacturing Resources Planning}

The successor of MRP is called Manufacturing Resources Planning (MRP II), which acts as an extension to MRP and largely deals with most of its problems. MRP II is used for effective planning of all kinds of manufacturing resources, including human resources. It addresses operational planning in units, financial planning and even incorporates simulation capability to answer "what-if" questions. In contrast to its predecessor, it can use both finite and infinite capacity planning. Fluctuations in forecast data are taken into account by including simulation of the MPS, thus creating a long-term control. Enterprise Resource Planning (ERP) systems could eventually be seen as an evolution of MRP II.

\subsection{Drum-Buffer-Rope}

Drum-Buffer-Rope (DBR) is a manufacturing execution methodology that is derived from the theory of constraints. It is classified as pull system and based on the assumption that there are a limited number of scarce resources which define the overall output of the manufacturing plant (Jodlbauer \& Huber, 2008). Basically, it consists of three key elements: the drum, the buffer, and the rope. 
The drum is the physical constraint of the factory and represents the element that limits the ability of the entire system to produce more. The rest of the manufacturing plant follows the beat of the drum and makes sure that the drum always has enough work and that anything it has processed does not get wasted. The buffer protects the drum by ensuring it always has work flowing to it. Buffers in DBR have time as their unit of measure, rather than quantity of material. This causes the priority system to strictly operate based on the time an order is expected to arrive at the drum. The rope acts as the release mechanism for the manufacturing plant. It depends on the progress of the drum and releases orders once the drum has finished a certain amount of work.

\subsection{Kanban}

The concept of Kanban is tightly related to lean manufacturing (LM) and just-intime (JIT) production. Basically, the focus of LM is on preserving value with less work. The expenditure of resources for any goal other than the creation of value for the customer is considered to be wasteful, thus target for elimination. The reduction of lead time is an important goal of LM. JIT is an essential pillar of LM and strives to improve the return-of-investment of a business by reduction of WIP and associated carrying costs. This means that manufacturing only takes place when necessary.

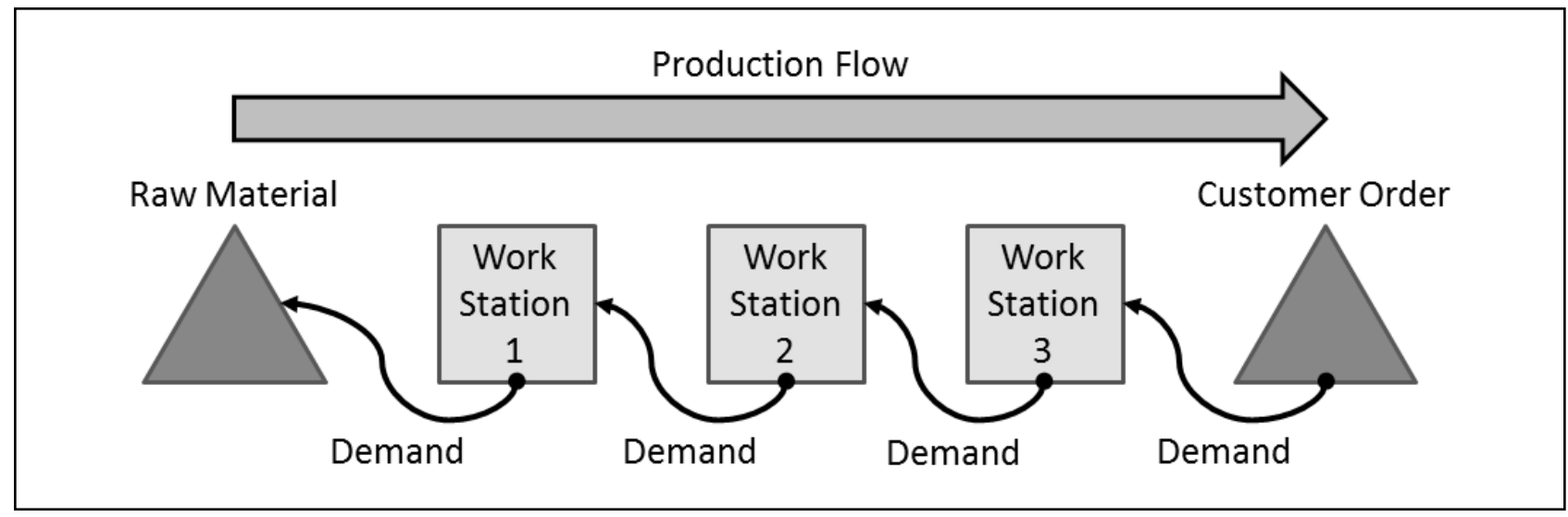

Fig. 2. In Kanban systems, demand is generated between manufacturing steps

Kanban is a pull-based production control and scheduling system. It utilizes physical authorization cards (so-called Kanbans) that help to create a demand-driven system by signalling depletion of components or products between two workstations of the production chain (see figure 2). As soon as such a signal is received by a workstation, a process to replenish the goods at the subsequent workstation is triggered. By using a fixed amount of cards, WIP at each manufacturing stage is tightly controlled and limited to the total amount of cards used between two workstations (Marek et al., 2001). Individual card sets are used at different workstations. This creates individual demand at each precedent workstation.

Due to the use of physical cards, the concept is typically implemented as a manual system. However, the transition to electronic Kanban systems becomes more and more common. Electronic implementations have several advantages when compared to their manual counterparts. For example, they eliminate problems such as lost cards or manual input errors. 


\section{Constant Work-In-Process}

Spearman, Woodruff and Hopp presented Constant Work-In-Process (CONWIP), which is still relatively unknown in Europe, as an enhanced and generalized form of Kanban (Spearman et al., 1990). Its basic notion is to ensure a constant level of WIP throughout the whole system. Compared to Kanban, however, it is not a pure pull system but also incorporates aspects of push systems (Jodlbauer, 2008). CONWIP extends the advantages of Kanban's demand-driven production with the push approach of MRP. While Kanban uses individual card sets between each pair of workstations, only a single global set of cards is used for the whole production process in CONWIP (see figure 3) (Jodlbauer \& Huber, 2008).

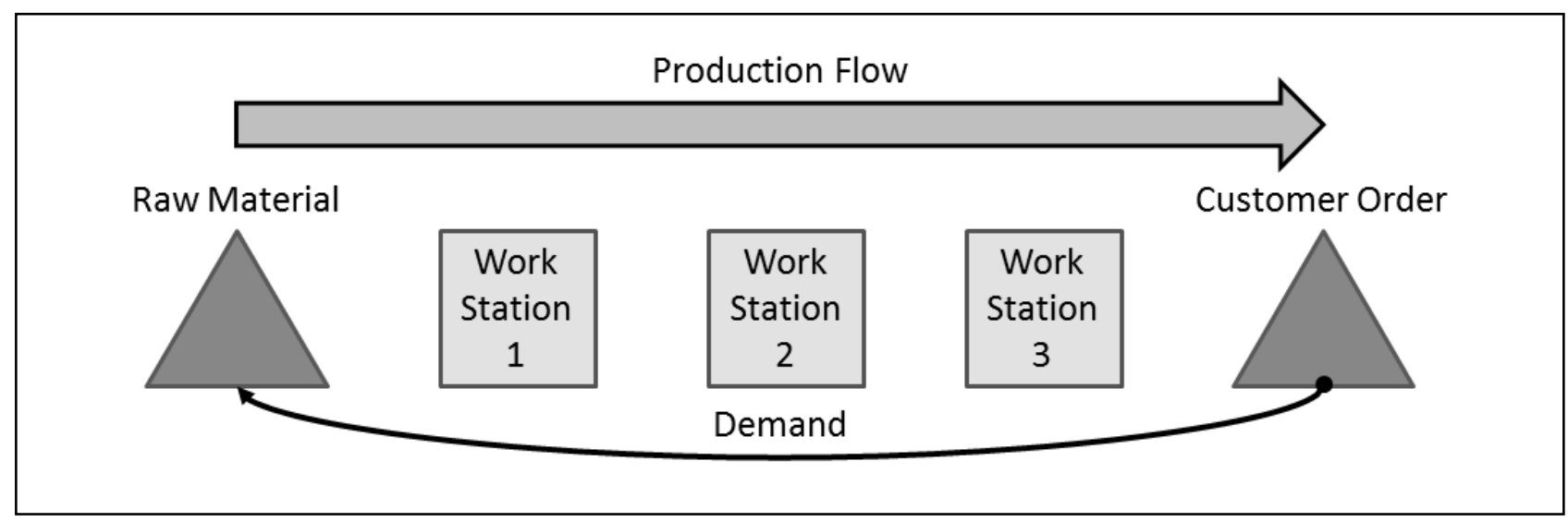

Fig. 3. In CONWIP systems, actual demand from the customer triggers the release of new work items to the production line

In its most simple form, CONWIP is a list-based pull system where demand triggers the release of new equivalently-sized work units for production. Each of these is assigned a global authorization card from the card set that remains associated to that work unit until the manufacturing process for this element is complete. Once released, each work unit is pushed through the manufacturing chain until the final product leaves production. At that point, its associated authorization card is released, which allows a new work unit to enter the manufacturing system. With this approach, WIP is not only controlled for each production step but for the whole production system. WIP remains constant (thus the name of CONWIP) as the total amount of cards within the manufacturing system is also stable. In case of a bottleneck, CONWIP allows to reduce the total number of cards. On the other hand, it is also allowed to increase the number of cards in order to raise WIP and to ensure higher throughput (Marek et al., 2001).

\subsection{Aspects for Small- and Medium-Sized Businesses}

The implementation of CONWIP in production systems brings several advantages, which may be especially important for small- and medium-sized businesses: Flow times of CONWIP systems are easily predictable due to constant WIP levels (Spearman et al., 1990). Hence, delivery reliability is also increased (Altendorfer \& Jodlbauer, 2007). Furthermore, CONWIP supports prioritization of 
production orders and allows for MTO production even if many variants and materials are used.

When compared to Kanban, CONWIP production systems are easier to manage because there is only a single global set of cards that has to be adjusted for the whole system (Marek et al., 2001). According to (Enns \& Rogers, 2008), however, it is hard to compare the actual performance of CONWIP with that of other systems like Kanban or MRP. It was found that different studies came to varying conclusions in regard to performance of these systems, as seen in (Altendorfer \& Jodlbauer, 2007), (Enns \& Rogers, 2008), (Hochreiter, 1999), and (Jodlbauer \& Huber, 2008). Therefore, it is not feasible to provide a general recommendation on which manufacturing system to use.

Aspects for small- and medium-sized companies have previously been discussed in an earlier paper (Gastermann et al., 2011), because of which this paper will not cover this topic in any more detail.

\subsection{Practical List-Based Production Planning}

Generally, CONWIP is quite a simple approach without much overhead. It is easy enough to work with even for unskilled staff. Basically, the simplest way to perform production planning in CONWIP systems is by means of a production order list (Altendorfer \& Jodlbauer, 2007). With such a list it is possible to plan and trigger the release of production orders to the production line. The list is filled with work orders by a MPS system that acts independently from the production control system. Each item within the list represents a single order. These work orders are then processed sequentially by the manufacturing system. The arrangement and the release of orders to the production line are influenced by various parameters (Altendorfer \& Jodlbauer, 2007), which will be discussed in the upcoming paragraphs:

The work-ahead-window (WAW) is a timeframe in which work orders are scheduled and released for production (see figure 4, indices 2 to 11). However, they are only scheduled or released if their due dates lie within the timeframe of the WAW. The purpose is to avoid that too much work is released in low-selling periods. The system is thereby able to automatically reduce WIP and output quantity to the level of actual demand. With CONWIP, it is not desirable to move known production orders forward or to switch over to MTS production in order to bridge the time of a low-selling period. The reason is that it is always possible for customers to change or cancel orders at short notice. The effect of automatic WIP reduction could decrease if the WAW is set too high, but could also result in poor delivery reliability if its timeframe is set too low.

The capacity trigger describes the maximum amount of work the production line can handle within a certain timeframe without the allocation of additional working resources (like overtime or extra shifts, for example). Consequently, this parameter helps to detect capacity bottlenecks. The capacity trigger supervises the total amount of work within the WAW, which is a combination of WIP and the amount of scheduled work (see figure 4 , indices 2 to 11 ).

The dispatching rule determines the sequential arrangement in which scheduled items are released for production (see figure 4, indices 7 to 11). By default, the rule is 
based on the date of delivery (Spearman et al., 1990). In this case, the scheduled work order with the earliest delivery date would be released next. In figure 4, for example, this would be the item at index 7. However, it is also possible to use different dispatching rules. Similarly, the processing rule determines the order in which released items are processed within production (see figure 4, indices 2 to 6). Although these two rules determine the arrangement of items within the list, it is always possible to manually overrule the suggested order and prioritize certain production orders, even if it would have negative effect on due dates of other items. The degree of influence on other production orders depends on actual workload and the target date of the prioritized work item.

\begin{tabular}{|c|c|c|c|c|c|c|c|c|c|c|}
\hline \multicolumn{2}{|c|}{ Index } & \multicolumn{3}{|c|}{ Amount } & Status & Target Date & \multicolumn{4}{|c|}{ Other Data } \\
\hline & 1 & \multicolumn{3}{|l|}{940} & completed & 13.10.2012 & \multicolumn{3}{|l|}{$\ldots$} & \\
\hline & 2 & 5400 & \multirow{5}{*}{ 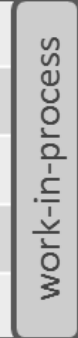 } & \multirow{10}{*}{ 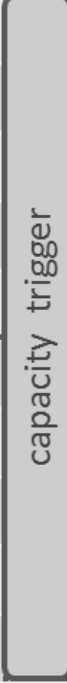 } & in production & 13.10.2012 & $\ldots$ & \multirow{10}{*}{$\begin{array}{l}3 \\
0 \\
\frac{0}{0} \\
\frac{5}{3} \\
\frac{1}{0} \\
\frac{\pi}{0} \\
\frac{1}{\pi} \\
\frac{1}{2} \\
\frac{1}{3} \\
3\end{array}$} & \multirow{5}{*}{ 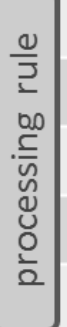 } & \\
\hline & 3 & 1750 & & & in production & 16.10.2012 & $\ldots$ & & & \\
\hline & 4 & 3200 & & & in production & 17.10 .2012 & $\ldots$ & & & \\
\hline & 5 & 7000 & & & in production & 17.10.2012 & $\ldots$ & & & \\
\hline & 6 & 2550 & & & in production & 21.10 .2012 & $\ldots$ & & & \\
\hline I & 7 & 4750 & & & scheduled & 23.10.2012 & $\cdots$ & & \multirow{5}{*}{ 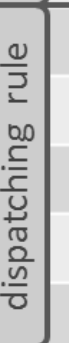 } & | \\
\hline I & 8 & 6500 & & & scheduled & 25.10 .2012 & $\cdots$ & & & I \\
\hline I & 9 & \multicolumn{2}{|l|}{1500} & & scheduled & 25.10 .2012 & $\ldots$ & & & i \\
\hline I & 10 & \multicolumn{2}{|l|}{8775} & & scheduled & 02.11 .2012 & \multirow{2}{*}{$\begin{array}{l}\ldots \\
\ldots\end{array}$} & & & i \\
\hline | & 11 & 2100 & & & scheduled & 06.11 .2012 & & & & I \\
\hline & 12. & \multicolumn{3}{|l|}{4120} & pending & 07.11 .2012 & & & & \\
\hline
\end{tabular}

Fig. 4. This is a simplified example of a CONWIP production planning list. Vertical bars mark the scope of relevant list parameters

At last, the WIP cap defines the maximum amount of work on which the production system is allowed to concurrently work on (see figure 4, indices 2 to 6 ). This parameter prohibits the release of new work as long as the combination of current WIP and work content of the new order exceed the currently set WIP cap. The value for this parameter is usually determined by the bottleneck of the production line. It should be set to a value that ensures that the bottleneck never runs out of material, even in disadvantageous and unexpected situations. A reduction of the WIP cap would consequently reduce both WIP and lead time of production. However, if set too low it could also cause negative effect on output quantity and delivery reliability of the system.

Figure 4 depicts a simplified CONWIP production control list and shows the scope of all of the previously discussed list parameters. Each work order that is added to this list is required to contain at least information about the amount of work it takes as well as its target date. Based upon these two values the CONWIP list can be 
generated. The list is divided into four sections, each containing work items of a different status:

The topmost section (see index 1 in figure 4) contains production orders whose status is "completed". Each of these items have already run through production and are now available on stock as finished products in required quantity. Completed work is no longer considered for production planning.

The second section (see indices 2 to 6 in figure 4) groups work orders that are currently being processed on the production line, thus having the status "in production". The total amount of WIP in this group must not exceed the specified WIP cap. The sequence in which these items are processed is determined by the processing rule.

All other items within the WAW that would be ready for production but cannot be started yet because of the WIP cap are placed in the third section of the list (see indices 7 to 11 in figure 4). Their status is "scheduled" because work orders are allowed to enter production as soon as a currently processed item is finished and the difference between WIP and WIP cap is enough for the new item to fit. The order of work units within this status group is determined by the dispatching rule.

Production orders that are not yet captured by the WAW have a "pending" status as they are still awaiting the date of scheduling. The group of pending work items forms the last section (see index 12 in figure 4) of the CONWIP planning list.

\section{Adopting CONWIP in Plastics Industry}

In this section, focus is on a recently conducted case study in which CONWIP has been implemented for evaluation in a separated area of a manufacturing plant. The plant is part of a medium-sized manufacturing company operating in the sanitary branch of plastics industry. Motivation to perform such a case study is based on the fact that said company did not incorporate an efficient PPC system. Until then, manufacturing planning had been carried out just by manual methods. Although that approach may work under certain circumstances, it became necessary to incorporate an approved and more effective PPC system in order to keep up with competition and increasing demand. At first, requirements had to be specified. The target was to apply a production system that is transparent, easy to manage, and highly efficient for the respective type of production. Classical ERP solutions, for example, provide an extensive range of functionality, but are therefore also mostly afflicted with high complexity. However, high complexity for production planning was not a desirable objective, so other production strategies had to be assessed as well. In the end, the decision to make use of a hybrid production strategy that incorporates ROP and CONWIP as planning technologies has been made because of the inherent advantages of such a manufacturing system.

Figure 5 illustrates the new composition of the implemented production process. It is inspired by a typical hybrid production approach that is divided into two manufacturing phases. The first phase is handled like a classical MTS production system. In this stage, raw material is processed by plastic moulding presses that produce generalised components based on ROP planning. These components are then 
stocked on a buffer, which acts as an intermediate storage for semi-finished products. This buffer represents the CODP of the hybrid system. Whenever its stock level drops below a certain level, production order are automatically created that initiate the process to produce missing components and refill the buffer.

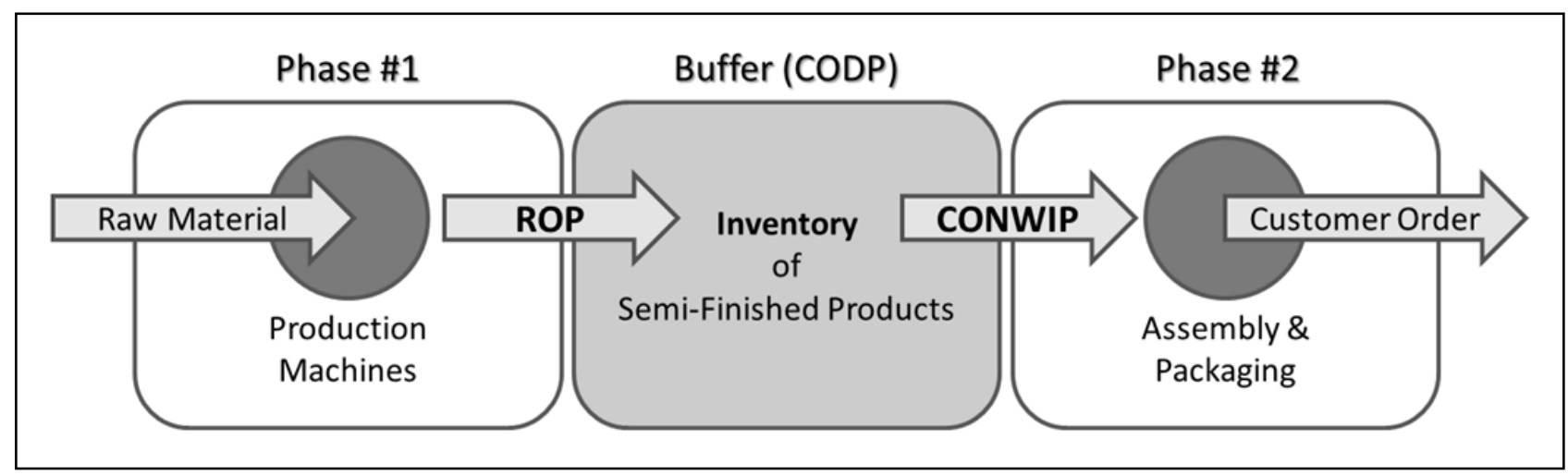

Fig. 5. This schematic representation shows the new hybrid production process

The second phase is realized by means of an MTO approach. Here, CONWIP is used for planning and control. As soon as a customer order is placed, an internal assembly and packaging order is created. This order is then scheduled by a CONWIP planning tool, which could be a simple order list as described in the previous section. Components are taken from the buffer for each released order and a specific product variant is built. Eventually, the finished products of the order are packaged and sent to the customer. Unlike in the first phase, assembly and packaging processes are only initiated by actual demand, that is, the placement of customer orders.

However, difficulties arise from the fact that CONWIP is not a commonly used approach for production planning. While it is usually no problem to use an existing ERP system (which would be required anyway) for ROP planning, CONWIP-based planning is typically not supported by ERP systems in most cases. It would therefore be necessary to develop a proprietary solution that is able to do this.

\section{Conclusion}

The case study presented in this paper demonstrated the implementation of CONWIP in real production and highlighted a significant drawback: The lack of planning tools that actually support CONWIP. In order to resolve this problem, a customized solution has to be found. In this particular case, it was decided to develop a custom planning application with which it would be possible to schedule internal orders based on CONWIP principles. The advantage of this is that such an application would be highly specialised for specific use cases and thus easier to use by staff than conventional ERP systems.

Segmentation of production processes, as seen by the realized hybrid production system, is an important aspect in order to deal with increasing pressure from global competition. In order to satisfy requirements from both customers and economy, it is vital to lower production costs as well as to increase flexibility. The presented system is one approach on how to achieve this. It is able to reduce WIP, but also able to 
increase flexibility and throughput by use of a buffer. Beyond that, a buffer also allows for easier variant management because consumer-specific variants are not built until the end of the manufacturing process.

Future research is planned on the topic of the proprietary CONWIP-based planning application that is required to manage the second stage of the presented production process. It is intended that a subsequent paper will return to this topic to discuss, among others, aspects of implementation and use cases of this application in more detail. In this context, calibration of CONWIP list parameters should also be described. Another area of further research would be to evaluate long-term reliability of the presented system and compare it to implementations of other companies.

\section{References}

Altendorfer, K. \& Jodlbauer, H. (2007). CONWIP - Hohe Liefertreue bei gleichzeitig niedrigen Beständen, In: PPS Management, Vol. 2007, No. 1, Gronau, N., (Ed.), pp. 16-19, Gito, ISBN 9783940019011, Berlin

Enns, S. T. \& Rogers, P. (2008). Clarifying CONWIP versus Push System Behavior using Simulation, Proceedings of the 2008 Winter Simulation Conference, Mason, S. J., (Ed.), pp. 1867-1872, ISBN 9781424427086, Miami, December 2008, Miami

Gastermann, B. C.; Stopper, M. \& Katalinic, B. (2011). Aspects of the Production Control System CONWIP in Small and Medium Sized Industrial Companies, Annals of DAAAM for 2011 \& Proceedings of the 22nd International DAAAM Symposium, Katalinic, B., (Ed.), pp. 621-622, ISBN 9783901509834, ISSN 1726-9679, DAAAM International, Vienna, Austria, 2011

Hochreiter, T. A. (1999). A Comparative Simulation Study Of Kanban, CONWIP, and MRP Manufacturing Control Systems in a Flowshop, MSc. Thesis, Dept. Industrial \& Systems Engineering, University of Florida, Gainesville

Jodlbauer, H. (2008). Produktionsoptimierung: Wertschaffende sowie kundenorientierte Planung und Steuerung, Springer, ISBN 9783211781401, Vienna

Jodlbauer, H. \& Huber, A. (2008). Service level performance of MRP, KANBAN, CONWIP and DBR due to parameter stability and environmental robustness, International Journal of Production Research, Vol. 46, No. 8, (April 2008) pp. 2179-2195, ISSN 0020-7543

Marek, R. P.; Elkins, D. A. \& Smith, D. R. (2001). Understanding the Fundamentals of Kanban and CONWIP Pull Systems using Simulation, Proceedings of the 2001 Winter Simulation Conference, Peters, B.A., (Ed.), pp. 921-929, ISBN 9780780373075, Arlington, IEEE Computer Society, Washington

Olhager, J. (2003). Strategic Positioning of the Order Penetration Point. International Journal of Production Economics, Vol. 85, No. 3, (September 2003) pp. 319329, ISSN 0925-5273

Spearman, M. L.; Woodruff, D. L. \& Hopp, W. J. (1990). CONWIP: a Pull Alternative to Kanban. International Journal of Production Research, Vol. 28, No. 5, (May 1990) pp. 879-894, ISSN 0020-7543 